\title{
Folate status and mood: is there a relationship?
}

\author{
Emma Williams ${ }^{1}$, Barbara Stewart-Knox ${ }^{1, *}$, Chris McConville ${ }^{2}$, lan Bradbury ${ }^{3}$, \\ Nicola C Armstrong ${ }^{1}$ and Helene McNulty ${ }^{1}$ \\ ${ }^{1}$ Northern Ireland Centre for Food and Health (NICHE), University of Ulster, Coleraine BT52 1SA, Northern \\ Ireland: ${ }^{2}$ School of Psychology, University of Ulster, Coleraine, Northern Ireland: ${ }^{3}$ School of Biomedical \\ Sciences, University of Ulster, Coleraine, Northern Ireland
}

Submitted 17 May 2006: Accepted 30 November 2006: First published online 13 June 2007

\begin{abstract}
Objective: To explore the relationship between subjective mood, folate status and homocysteine in healthy individuals.

Design: Subjective mood assessments were completed twice daily over the course of one week using the Positive and Negative Affect Scale (PANAS). The PANAS is a validated scale which considers mood state on two distinct continua, one reflecting positive mood and the other negative mood, each requiring response to 10 adjectives on a Likert scale. A blood sample was taken on one occasion at the start of the week during which subjective mood was assessed and analysed for red-blood-cell (RBC) folate, serum folate and plasma homocysteine concentrations.

Subjects: Male volunteers aged $19-47$ years $(n=58)$ were recruited from local industries.

Results: High concentrations of RBC folate were associated with less variability (lower standard deviation) in negative mood $(P=0.023)$. Subjective mood, however, was not related to serum folate or homocysteine.

Conclusions: This study appears to be the first to uncover an association between long-term folate status and subjective mood (employing the PANAS) in healthy males. More research is needed to further explore the relationship between nutritional status and mood.
\end{abstract}

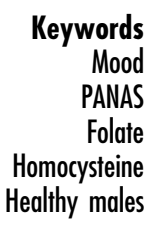

Mental health problems account for an increasingly high proportion of adult morbidity in the UK, which, along with nutrition, is high up on the list of public health policy priorities, with the emphasis on prevention ${ }^{1}$. There is therefore a continuing need for research into links between psychological well-being and nutritional factors $^{2}$, especially given that nutritional status can be amenable to intervention and improvement. Mood may be dependent, at least in part, upon nutritional factors. Evidence from clinical samples has implied that up to a third of psychiatric patients with depression may have suboptimal folate status ${ }^{3,4}$. Individuals with major depression have consistently been shown to have lower serum or red-blood-cell (RBC) folate concentrations ${ }^{4-6}$, deficiency of which can be associated with more pronounced symptoms ${ }^{3,6,7}$. There is some evidence to suggest that increased folate intake can improve mood in the clinically depressed ${ }^{8,9}$. Folate and vitamin $\mathrm{B}_{12}$ status may also determine the response of clinically depressed individuals to treatment with antidepressant medications ${ }^{10}$.

A potential interdependent relationship may exist between folate status, homocysteine and mood. Folate and vitamin $\mathrm{B}_{12}$ are both co-factors involved in the remethylation of homocysteine to methionine. High levels of homocysteine are associated with an increased risk of cardiovascular disease ${ }^{11}$, neurodegenerative disorder $^{12}$ and therefore possibly mood. Methylation is dependent upon levels of the major methyl donor $S$-adenosylmethionine (SAM). SAM in turn is derived from the methionine cycle in the presence of folate in the form 5-methyltetrahydrofolate and vitamin $\mathrm{B}_{12}$ in the form of methylcobalamin. SAM is required for transmethylation reactions in the central nervous system ${ }^{13,14}$ and has been shown to increase cerebrospinal fluid levels of 5-hydroxyindoleacetic acid (5-HIAA) in rats $^{15}$ and humans with clinical depression ${ }^{16}$. 5-HIAA is also the main metabolite for the monoamine neurotransmitter serotonin (5-hydroxytryptamine; $5-\mathrm{HT}$ ), the synthesis ${ }^{17}$ and activity ${ }^{18-20}$ of which have been found lacking in clinically depressed individuals. In addition, folate deficiency has been associated with decreased 5-HT activity in clinically depressed individuals ${ }^{8}$ which appears to recover in response to folic acid supplementation ${ }^{21}$. Low folate status/intake can lead to increased homocysteine concentrations ${ }^{22}$ and 
reduced 5-HT activity which may have implications for $\operatorname{mood}^{23}$.

Plasma homocysteine increases as a consequence of suboptimal folate status and declines in response to supplemental folic $\operatorname{acid}^{24}$. Individuals homozygous for the $677 \mathrm{C} \rightarrow \mathrm{T}$ polymorphism (TT genotype) in the gene encoding methylenetetrahydrofolate reductase (MTHFR) may typically have elevated homocysteine levels ${ }^{25}$ and lower folate status ${ }^{26}$. Epidemiological research, carried out as part of the British Women's Heart and Health Study, has indicated that women with this polymorphism have a higher incidence of depression than women without $\mathrm{it}^{27}$. This adds further evidence to the notion that high levels of homocysteine could be detrimental to psychological well-being in this genotype ${ }^{28,29}$. Little non-epidemiological research appears to have considered the potential impact of folate and homocysteine upon the mood of healthy individuals who do not have the MTHFR $677 \mathrm{C} \rightarrow \mathrm{T}$ polymorphism.

There is an apparent dearth of research into the role of folate in the regulation of mood in healthy individuals. What little research exists relating to healthy populations has provided no evidence for an association between folate or vitamin $\mathrm{B}_{12}$ deficiency and depressed mood (as assessed by the Beck Depression Inventory) ${ }^{30}$ or any alteration in mood in response to either folate or vitamin $\mathrm{B}_{12}$ in older females ${ }^{31}$ or in response to folic acid in young males ${ }^{32}$. The present study meets a need for further prospective research into the relationship between folate status and mood in healthy individuals. The aim was to assess the relationship between subjective mood, folate status and homocysteine in a group of healthy male volunteers from which those with the MTHFR $677 \mathrm{C} \rightarrow \mathrm{T}$ polymorphism have been excluded.

\section{Method}

Ethical approval was obtained from the Research Ethical Committee of the University of Ulster. The study was of a correlational design, exploring the relationships between subjective mood, folate status and plasma homocysteine.

\section{Sampling}

Healthy males $(n=65)$ were recruited during July and August 1999, from amongst university staff/students and employees in local industries, for screening and potential recruitment to subsequent nutritional intervention studies. Recruitment was via letters of introduction, poster advertisements and attendance at public/group meetings. The $677 \mathrm{C} \rightarrow \mathrm{T}$ polymorphism in MTHFR can be associated with impaired folate metabolism ${ }^{33}$ and increased risk of depression ${ }^{30,34}$. Given that the research aimed to study healthy individuals, volunteers were genotyped and those found to have the homozygous mutant TT genotype at screening were excluded from the study sample. Those who reported taking supplements containing folic acid or other B-vitamins, and/or consuming folic acid-fortified foods such as breakfast cereals, or taking medication known to impair folate status, were also excluded. Other exclusion criteria included those reporting depression or diseases known to impact upon folate status including gastrointestinal conditions, haematological disorders and vascular, hepatic or renal disease.

\section{Sample description}

A total of 58 healthy males aged 19-47 years with an average age of 30 years took part in this investigation. In terms of social class ${ }^{35}, 15.4 \%$ were in managerial/ professional roles, 29.2\% were semi-professional, 24.6\% were non-manual skilled workers, $4.6 \%$ were manually skilled workers and 26.2\% were unskilled.

\section{Procedure}

Participation in the study required written informed consent. Volunteers were required to give one single fasting blood sample, which was subsequently analysed for RBC folate, serum folate and homocysteine, at the commencement of the same week in which subjective mood was assessed. Mood was recorded using the Positive and Negative Affect Schedule (PANAS) twice daily ( 6 and $12 \mathrm{~h}$ after waking), at approximately 12.30 and 19.00 hours, for a period of one week. The period of a 'week' was assumed to encompass both work and leisure time $^{36}$ as well as day-to-day variation in mood across the course of the week ${ }^{37}$. Mood was therefore assessed on 14 separate occasions. At each time point at which mood was assessed, volunteers were required to respond to 20 adjectives, half of which related to positive mood and half to negative mood, on a 5 -point Likert scale $(1=$ very slightly to $5=$ extremely).

\section{Blood sampling and analysis}

A (12-h) fasting blood sample was collected between 08.00 and 09.00 hours by a qualified phlebotomist. The sample $(19.5 \mathrm{ml})$ was collected by venepuncture and deposited into pre-evacuated tubes coated with ethylenediaminetetraacetic acid $(1 \times 8 \mathrm{ml}$ and $1 \times 4.5 \mathrm{ml})$ and analysed for a full blood picture/complete blood count, RBC folate and plasma homocysteine. A sample was also deposited into a separate $7 \mathrm{ml}$ Vacuette Serum-Separator Tube (Greiner Labortechnik) for analysis of serum folate. Post-collection, all blood samples were prepared, kept on ice and fractionated within $1-2 \mathrm{~h}$ of collection, and then stored at $-70^{\circ} \mathrm{C}$ until batch analysis within 6 months of sampling. The $M T H F R$ 677C $\rightarrow$ T genotype was identified by amplification with polymerase chain reaction followed by digestion with HinF1 restriction enzyme (Gibco Life Technologies) ${ }^{26}$. DNA was extracted from fasting frozen whole blood samples by incubating with proteinase with 
the use of the QIAamp DNA Blood Mini Kit (QIAGEN Ltd). Plasma homocysteine was measured by the Abbott IMx fluorescence polarisation immunoassay ${ }^{38}$. Serum and RBC folate were assessed using the Lactobacillus case $i$ microbiological assay ${ }^{33}$. Blood was analysed for a full blood picture/complete blood count using an automated Coulter counter (Mater Hospital, Belfast, Northern Ireland).

\section{Subjective mood assessment}

The PANAS, a well-validated measure developed by Watson et al. in 1988, was used to determine subjective $\operatorname{mood}^{39}$. The PANAS, which provides a measure of mood state at the time of testing, defines mood as 'a transient episode of feeling or affect ${ }^{, 40}$ (p. 4). This self-administered scale measures two broad distinct dimensions of 'affect'. Positive affect (PA) refers to the extent to which a person feels enthusiastic, active or alert. Low PA implies sluggishness. Negative affect (NA) refers to a general dimension of subjective distress, encompassing a variety of aversive mood states such as anger, contempt, disgust, fear and nervousness. Low NA implies calmness and serenity ${ }^{39}$. That PANAS scores have been found to correlate with wholeblood serotonin levels in healthy males ${ }^{41}$ and healthy postmenopausal women ${ }^{42}$ suggests that it could provide a sensitive tool with which to study mood in relation to other potentially mood-related biochemical indices.

\section{Statistical analysis}

Statistical analysis was performed using the Statistical Package for the Social Sciences, version 11.0 (SPSS Inc.). Univariate linear regression was employed to determine associations between subjective mood and blood biochemical measurements. Mood (PA and NA; PA variability and NA variability) was the dependent variable and each of the biochemical measures (RBC and serum folate, plasma homocysteine) were the independent variables. Given that each scale measures a distinct dimension of subjective mood, the regression analysis was performed separately for each measure of affect against each biochemical measure. No differences were observed in mood ratings by time of day or day of the week. Mean scores for positive and negative mood and mood variability were therefore summed over time of day and day of the week for each individual and entered into the analysis. Subjective mood was analysed using the mean of each individual's score for positive and negative affect, while variability in subjective mood was calculated from the mean standard deviation (SD) of each individual's positive and negative affect (PANAS) score, which was entered into the analysis. Each individual was therefore assigned a score summarising responses across adjectives representing positive mood, negative mood and the variability in both positive and negative mood. Internal consistency reliability was tested using Cronbach's $\alpha$ coefficient, a stability coefficient for behavioural measures $^{43}$. Both the PA and NA scales were found to have high reliability (standardised reliability coefficient, $\alpha=0.96$ and $\alpha=0.93$ respectively).

\section{Results}

\section{RBC folate and mood}

Mean ( $\pm \mathrm{SD})$ RBC folate concentration was $1046 \pm 665 \mathrm{nmoll}^{-1}$, which is at the higher end of the normal range (150-1000 nmoll ${ }^{-1}$ ) (Department of Medicine, Trinity College Dublin). Of the sample of 58 individuals, only three could be considered folate-deficient $\left(\leq 100 \mathrm{nmoll}^{-1}\right)$. Mean $( \pm \mathrm{SD})$ values for affect were as follows: PA, $25.41 \pm 5.85$; NA, $12.72 \pm 2.69$; PA variability, $9.13 \pm 1.93$; NA variability, $5.43 \pm 2.66$. As RBC folate increased, NA variability decreased $(F(1,56)=5.491$, $P=0.023)$ (Fig. 1). RBC folate was not associated with PA ( $P=0.491)$ or NA $(P=0.325)$ or the degree of PA variability $(P=0.361)$.

\section{Serum folate and mood}

Mean ( \pm SD) serum folate concentration was $9.8 \pm 6.7 \mathrm{nmoll}^{-1}$, which is within the normal range of 2.7-20 nmoll ${ }^{-1}$ (Vitamin Research Laboratory, Trinity College Dublin). No associations were evident between serum folate and PA $(P=0.272)$ or NA $(P=0.841)$ or with variability in PA $(P=0.133)$ or NA $(P=0.992)$.

\section{Plasma bomocysteine and mood}

Mean ( \pm SD) plasma homocysteine concentration was $11.9 \pm 3.0 \mathrm{moll}^{-1}$. No relationships were evident between plasma homocysteine and PA $(P=0.305)$ and NA $(P=0.879)$ or with PA variability $(P=0.114)$ and NA variability $(P=0.759)$.

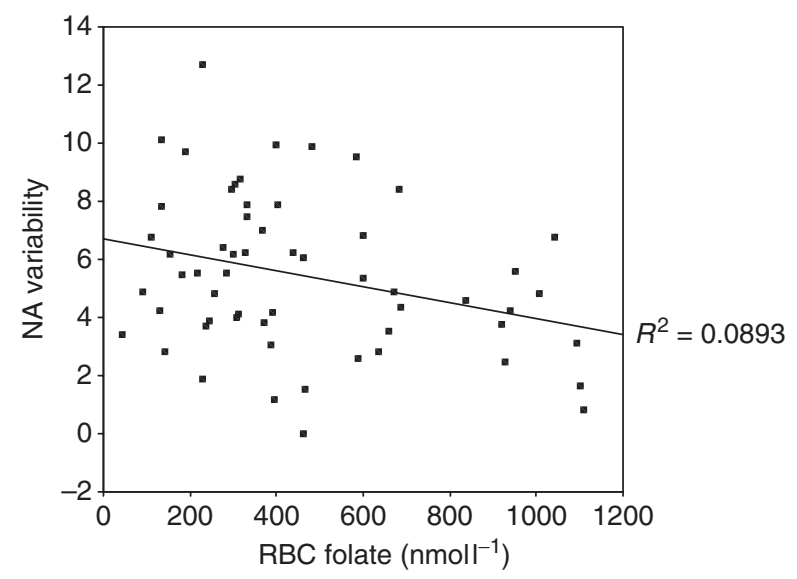

Fig. 1 Relationship between red-blood-cell $(\mathrm{RBC})$ folate and negative affect (NA) variability $(n=58)$ 


\section{Discussion}

The finding that lower RBC folate was associated with more labile negative mood in this group of healthy males is consistent with previous reports which have indicated that lower RBC folate concentrations can be related to depression in clinical populations ${ }^{4,44,45}$. The current findings and those of the aforementioned reports appear to contradict results from the few previous studies which have sampled healthy individuals and found little evidence that folate is associated with $\operatorname{mood}^{11}$ or benefits mood $^{12}$ in older women. The observation that RBC folate was related to the stability of negative mood is also difficult to equate with previous findings from a placebocontrolled intervention study previously carried out by our team (but employing a different sample), which suggested that folic acid, taken in physiological doses over a 3-month period, did not alter mood in healthy young males ${ }^{32}$. One possible explanation for the apparent discrepancy between these findings and those of our previous intervention study is that the intervention may have been of insufficient duration for a response in RBC folate - and therefore mood - to become evident. Of note, RBC folate is generally considered a more accurate reflection of long-term folate status than serum folate ${ }^{45}$. The observation that RBC folate, but not serum folate, was related to variability in negative mood in this study may imply that long-term folate status has implications for mood stability in non-clinical populations.

Mood variability is a stable individual characteristic ${ }^{46}$. It relates to how an individual's mood deviates from average levels to extremes (mood swings). Mood variability is thought to be important in the aetiology of non-clinical depressed states ${ }^{47}$. Previous research has shown evidence for a relationship between mood variability and depression in healthy individuals. McConville and Cooper ${ }^{48}$ obtained a positive correlation between mood variability using the PANAS and level of depression as defined by the Beck Depression Inventory ${ }^{49}$ and the Depression Adjective Checklist ${ }^{50}$ in a non-clinical healthy adult sample. Negative affect refers to a broad range of aversive mood states such as anger, contempt, disgust, fear and nervousness ${ }^{39}$. Increased variability in negative affect therefore may predispose certain individuals to a wide range of negative emotions. High negative affect and low positive affect are features of anxiety and depression respectively ${ }^{51}$. Our findings suggest that more labile negative mood could be related to negative mood states in healthy individuals.

Previous research provides evidence for a positive relationship between homocysteine and depression ${ }^{30,31}$, a relationship which appears to become stronger as plasma homocysteine levels increase. Contrary to what previous research would suggest, homocysteine was not found to be associated with mood in this sample of healthy males. Unlike these previous studies, however, individuals homozygous for the MTHFR $677 \mathrm{C} \rightarrow \mathrm{T}$ (TT) genotype were explicitly excluded from the current study at screening and, as a consequence, those with the highest homocysteine levels were excluded. This perhaps explains the lack of association between homocysteine and subjective mood. It is possible that the MTHFR genotype may interact with low folate status to impact deleteriously upon mood.

The finding that long-term folate status is related to mood stability is potentially important given that mild to moderate subclinical deficiencies of folate are believed to be commonplace within the general population ${ }^{52}$. Out of the 58 men sampled for this study, however, only three could be considered folate-deficient $\left(\leq 100 \mathrm{nmoll}^{-1}\right.$ ) while 10 had RBC folate level of $>800 \mathrm{nmoll}^{-1}$. The relationship observed between negative mood stability and RBC folate status was therefore not entirely linear. It is possible that if those individuals with the MTHFR $677 \mathrm{C} \rightarrow \mathrm{T}$ genotype had been retained in the study, the relationship may have been stronger. More research is needed on much larger cohorts where those homozygous for MTHFR $677 \mathrm{C} \rightarrow \mathrm{T}$ polymorphism are included, thus allowing the possibility for subgroup analysis, which would further elucidate not only the association between folate status and mood but the potential influence of genotype on the association. Meanwhile, these findings complement those of existing studies that have found relationships between homocysteine and mood in individuals of the TT genotype.

\section{Conclusion}

Previous research in this domain has focused primarily upon clinically depressed populations, often older individuals, who have been folate-deficient. This appears to be the first investigation of its kind to explore folate status and mood using the PANAS in healthy male volunteers. The exploratory nature of this study necessitates that conclusions remain tentative until further research is undertaken that controls for psychosocial and lifestyle factors and employs a larger, genetically heterogeneous sample of both males and females. Meanwhile, long-term maintenance of folate status may have possible benefits in the prevention of depression within the population as a whole. This may have implications for the current debate surrounding possible mandatory folic acid fortification of common foods such as bread ${ }^{53}$, which, although primarily aimed at preventing neural tube defects ${ }^{54}$, may hold other health benefits.

\section{Acknowledgements}

Sources of funding: These measurements were carried out as part of the Food Standards Agency project no. NO5013. 
The research was funded as part of a University of Ulster (Millennium)-funded PhD studentship.

Conflict of interest declaration: None.

Authorship contributions: E.W. carried out the fieldwork and co-authored the paper; B.S.-K. supervised the research and co-authored the paper; I.B. provided guidance on the statistical analysis; C.M. advised on mood measurement; N.C.A. facilitated the fieldwork; and H.M. advised on nutritional aspects of the research.

Acknowledgements: We gratefully acknowledge the contribution of Professor John Scott and Dr Anne Molloy, Departments of Biochemistry and Clinical Medicine, Trinity College Dublin, who carried out the homocysteine and folate analyses.

\section{References}

1 Wanless D. Securing Good Health for the Whole Population: Final Report. London: HM Treasury, 25 February 2004. Also available at http://www.hm-treasury.gov.uk/consultations_ and_legislation/wanless/consult_wanless04_final.cfm

2 Bodner LM, Wisner KL. Implications for improving mental health among childbearing-aged women. Biological Psychiatry 2005; 58: 679-85.

3 Reynolds EH, Preece JM, Bailey J, Coppen A. Folate deficiency in depressive illness. British Journal of Psychiatry 1970; 117: 287-92.

4 Carney MWP, Chary TKN, Laundy M, Bottiglieri T, Chanarin I, Reynolds EH, et al. Red cell folate concentrations in psychiatric patients. Journal of Affective Disorders 1990; 19: 207-13.

5 Ghadirian AM, Anath J, Engelsmann F. Folic acid deficiency in depression. Psychosomatics 1980; 21: 926-9.

6 Abouh-Saleh MT, Coppen A. Serum and red blood cell folate in depression. Acta Psychiatrica Scandinavica 1989; 80: $78-82$

7 Coppen A, Abouh-Saleh MT. Plasma folate and affective morbidity during long-term lithium therapy. British Journal of Psychiatry 1982; 141: 87-9.

8 Botez MI, Botez T, Levielle J, Biellmann P, Cadotte M. Neuropsychological correlates of folic acid deficiency: facts and hypotheses. In: Botez MI, Reynolds EH, eds. Folic Acid in Neurology, Psychiatry, and Internal Medicine. New York: Raven Press, 1979; 425-61.

9 Guaraldi G, Fava M, Mazzi F, LaGreca P. An open trial of methyltetrahydrofolate (MTHF) in elderly depressed patients. Annals of Clinical Psychiatry 1993; 5: 101.

10 Fava M, Borus JS, Alpert JE, Nierenberg AA, Rosenbaum JF, Bottiglieri T. Folate, vitamin $\mathrm{B}_{12}$, and homocysteine in major depressive disorder. American Journal of Psychiatry 1997; 154: 426-8.

11 Wald DS, Law M, Morris JK. Homocysteine and cardiovascular disease: evidence on causality from a meta-analysis. British Medical Journal 2002; 325: 1202-9.

12 Reynolds EH, Carney MWP. Methylation and mood. Lancet 1984; 2: 196-8.

13 Cantoni GL. S-adenosylmethionine: a new intermediate formed enzymatically from L-methionine and adenosine triphosphate. Journal of Biological Chemistry 1953; 204: 403-16.

14 Bottiglieri T, Hyland K, Reynolds EH. The clinical potential of ademethionine ( $S$-adenosylmethionine) in neurological disorders. Drugs 1994; 48: 137-52.

15 Ordonez LA, Wurtman RJ. Folic acid deficiency and methyl group metabolism in rat brain: effects of L-Dopa. Archives of Biochemistry and Biophysics 1974; 160: 372-6.
16 Agnoli A, Ruggieri S, Cerrone GG. The dopamine hypotheses of depression: results of treatment with dopaminergic drugs. In: Grattini S, ed. Depressive Disorders. Stuttgart: FK Schatauer Verlag, 1977; 447-58.

17 Lapin IP, Oxenkrug GF. Intensification of the central serotonergic processes as a possible determinant of the thymoleptic effect. Lancet $1969 ;$ 1: 132-6.

18 Le Quan-Bui KH, Plaisant O, Leboyer M, Gay C, Kamal L, Devynck $\mathrm{M}$, et al. Reduced platelet serotonin in depression. Psychiatry Research 1984; 13: 129-39.

19 Quintana J. Platelet serotonin and plasma tryptophan in endogenous depression. Clinical, therapeutic, and biological correlations. Journal of Affective Disorders 1992; 24: 55-62.

20 Coppen AP, Turner P, Rowsell AR, Padgham C. 5-Hydroxytryptamine (5-HT) in the whole blood of patients with depressive illness. Postgraduate Medical Journal 1956; 52: 156-8.

21 Botez MI, Young SN, Bachevalier J, Gauthier S. Effect of folic acid and vitamin $\mathrm{B}_{12}$ deficiencies on 5-hydroxindoleacetic acid in human cerebrospinal fluid. Annals of Neurology 1982; 12: 479-84.

22 Brattstrom L, Israellson B, Jeppson J, Hultberg B. Folic acid - an innocuous means to reduce plasma homocysteine. Scandinavian Journal of Clinical and Laboratory Investigation 1998; 48: 215-21.

23 Bottiglieri T. Homocysteine and folate metabolism in depression. Progress in Neuro-psychopharmacology and Biological Psychiatry 2005; 29: 1103-12.

24 Homocysteine Lowering Trialists Collaboration. Dose dependent effects of folic acid on blood concentrations of homocysteine: a meta-analysis of the randomized trials. American Journal of Clinical Nutrition 2005; 82: 806-12.

25 Frosst P, Blom HJ, Milos R, Goyette P, Sheppard CA, Mathews RG, et al. A candidate risk factor for vascular disease: a common mutation in methylenetetrahydrofolate reductase. Nature Genetics 1995; 10: 111-13.

26 Molloy AM, Daly S, Mills JL, Kirke PN, Whitehead AS, Ramsbottom D, et al. Thermolabile variant of 5,10methylenetetrahydrofolate reductase associated with low red-cell folates: implications for folate intake recommendations. Lancet 1997; 349: 1591-3.

27 Lewis SJ, Lawlor DA, Smith GD, Araya R, Timpson N, Day INM, et al. The thermolabile variant of MTHFR is associated with depression in the British Women's Heart and Health Study and a meta-analysis. Molecular Psychiatry 2006; 11: 352-60.

28 Bjelland I, Tell GS, Vollset SE, Refsum H, Ueland PM. Folate, vitamin $\mathrm{B}_{12}$, homocysteine, and the MTHFR 677C-T polymorphism in anxiety and depression: The Hordaland Homocysteine Study. Archives of General Psychiatry 2003; 60: 618-26.

29 Bottiglieri T, Laundy M, Crellin R, Toone BK. Homocysteine, folate, methylation, and monoamine metabolism in depression. Journal of Neurology and Psychiatry 2000; 69: 228-34.

30 Cassidy K, Kotynia-English R, Acres J, Flicker L, Lautenschlager NT, Almeida OP. Association between lifestyle factors and mental health measures among communitydwelling older women. Australian and New Zealand Journal of Psychiatry 2004; 38: 940-7.

31 Bryan J, Calvaresi E, Hughes D. Short-term folate, vitamin $\mathrm{B}_{12}$ or vitamin $\mathrm{B}_{6}$ supplementation slightly affects memory performance but not mood in women of various ages. Journal of Nutrition 2002; 132: 1345-56.

32 Williams E, Stewart-Knox B, Bradbury I, Rowland I, Pentieva K, Helander A, et al. Effect of folic acid supplementation and serotonin response in healthy males. British Journal of Nutrition 2005; 94: 602-8.

33 Molloy AM, Scott JM. Microbiological assay for serum, plasma, and red cell folate using cryopreserved, microtiter plate method. Methods in Enzymology 1997; 281: 43-53. 
34 Almeida OP, Flicker L, Lautenschlager NT, Leedman P, Vasikaran S, van Bockxmeer FM. Contribution of the MTHFR gene to the causal pathway for depression, anxiety and cognitive impairment in later life. Neurobiology of Aging 2005; 26: 251-7.

35 Office for National Statistics. The National Statistics SocioEconomic Classification User Manual. London: HMSO, 2002.

36 Farber ML. Time perspective and feeling tone a study in perception of the day. Journal of Psychology 1953; 35: $253-7$.

37 Larsen RJ, Kasimatis M. Individual differences in entrainment of mood to the weekly calendar. Journal of Personality and Social Psychology 1990; 58: 164-71.

38 Leino A. Fully automated measurement of total homocysteine in plasma and serum on the Abbott IMx Analyzer. Clinical Chemistry 1999; 45: 569-71.

39 Watson D, Clark LA, Tellegen A. Development and validation of brief measures of positive and negative affect: the PANAS scales. Journal of Personality and Social Psychology 1988; 54: 1063-70.

40 Watson D. Mood and Temperament. New York: The Guilford Press, 2000.

41 Williams E, Stewart-Knox B, Helander A, McConville C, Bradbury I, Rowland I. Associations between whole-blood serotonin and subjective mood in healthy male volunteers. Biological Psychology 2005; 71: 171-4.

42 Duffy ME, Stewart-Knox BJ, McConville C, Bradbury I, O'Connor $\mathrm{J}$, Helander A, et al. The relationship between whole-blood serotonin and subjective mood in apparently healthy postmenopausal women. Biological Psychology 2006; 73: $165-8$.

43 Nunnally JC. Psychometric Theory, 2nd ed. New Delhi: McGraw Hill Ltd, 1981.
44 Reynolds EH, Preece J, Johnson AL. Folate metabolism in epileptic and psychiatric patients. Journal of Neurology and Psychiatry 1971; 34: 726-32.

45 Godfrey PSA, Toone BK, Carney MWP, Flynn TG, Bottiglieri $\mathrm{T}$, Laundy $\mathrm{M}$, et al. Enhancement of recovery from psychiatric illness by methylfolate. Lancet 1990; 336: 392-5.

46 McConville C. Personality, motivational and situational influences on mood variability. Doctoral thesis, University of Ulster, Northern Ireland, 1992.

47 Costello J, Benjamin R, Angold A, Silver D. Mood variability in adolescents. A study of depressed, nondepressed and comorbid patients. Journal of Affective Disorders 1991; 23: 199-212.

48 McConville C, Cooper C. Mood variability and the intensity of depressive states. Current Psychology 1996; 14: 329-38.

49 Beck AT, Ward CH, Mendelsohn M, Mock J, Erbaugh J. An inventory for measuring depression. Archives of General Psychiatry 1961; 4: 561-71.

50 Lubin B. Depression Adjective Checklist. San Diego, CA: Educational and Industrial Testing Service, 1981.

51 Tellegen A. Structures of mood and personality and their relevance to assessing anxiety, with an emphasis on selfreport. In: Tuma $\mathrm{AH}$, Maser JD, eds. Anxiety and the Anxiety Disorders. Hillside, NJ: Erlbaum, 1985; 681-706.

52 Sauberlich HE. Relationship of vitamin B-6, vitamin B-12, and folate to neurological and neuropsychiatric disorders. In: Bendich A, Butterworth C, eds. Micronutrients in Health and Disease Prevention. New York: Marcel Dekker, 1991: 187-218.

53 Scientific Advisory Committee on Nutrition (SACN). Draft Report for Consultation: Folate and Disease Prevention. London: SACN, November 2005.

54 Wald N. Prevention of neural tube defects - results of the Medical Research Council vitamin study. Lancet 1991; 338: $131-7$. 